\title{
Vulval Lichen Sclerosus Associated with Vitiligo
}

\section{Rosa Giménez-García; Javier Cortejoso Hernandez}

Department of Dermatology, Hospital Rio Hortega, Calle Dulzaina 2, 47012 Valladolid. Spain rosagim@hotmail.com

** accepted as a poster in the 24 th EADV (European Academy of Dermatology and Venereology) Congress . Copenhague 7-11 October

Keywords: Vulvar lichen sclerosus; Vitiligo; Vulvar squamous, carcinoma

\section{INTRODUCTION}

Lichen sclerosus (LS) is an autoimmune, chronic inflammatory dermatosis with predilection for localized involvement of anogenital skin. Several lines of evidence support the hypothesis of an autoimmune basis for LS like a frequent association with autoimmune disorders. (1)We describe a 71 year-old patient with genital LS associated with vitiligo.

\section{CASE Report}

A 71-year-old woman presented to us with a 2 years history of genital pruritus. She had family history of vitiligo and thyroid disease, and personal history of vitiligo diagnosed 10 years ago. On physical examination the patient had depigmented macules on her left axillae, on her neck Fig 1)and on the pubic area consistent with vitiligo. Examination of the genital region revealed vitiligo patches on the pubis and scleroatrophic lesions on the vulva and perianal area (Fig 2). Histopathological study of a skin biopsy showed hyperkeratosis with parakeratosis, focal vacuolization of the basal layerand a dermal lichenoid infiltrate that was compatible with lichen sclerosus. Laboratory test including thyroid-stimulating hormone and autoantibodies were negative. She has been treated with pimecrolimus cream applied 2- 3 times per week

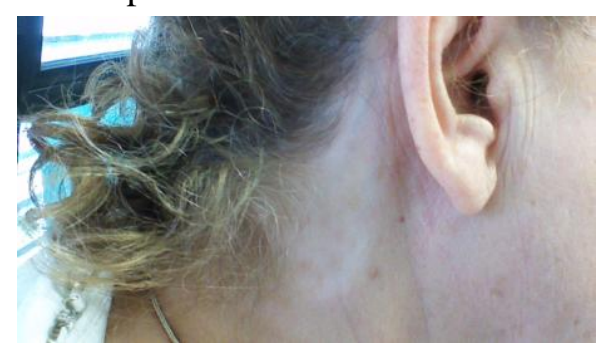

Fig1. Vitiligo on the neck

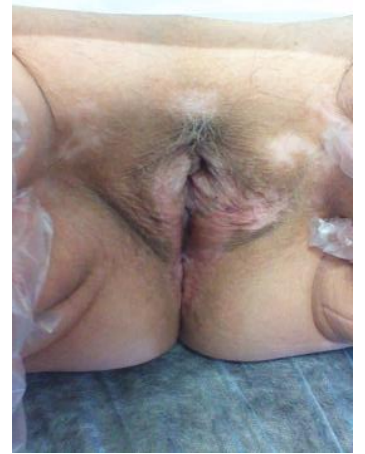

Fig2. depigmented macules on the pubic area consistent with vitiligo and scleroatrophic lesions on the vulva and perianal area 


\section{DISCUSSION}

The pathogenesis of LSA is thought to be a combination of environment and genetic factors. LS has a clear female preponderance and has two peak ages of presentation, in the prepuberal girls and boys and postmenopausal women and adult men(1). Association of LS with autoimmune diseases, especially thyroid disease, alopecia areata, morphea and pernicious anaemia, have been found $(2,3)$. In women, lichen sclerosus was significantly more often associated autoimmune thyroid disease, antithyroid-antibodes and elevated autoantibodies as compared to men. (2)

Vitiligo, a chronic systemic disease that mainly affects melanocytes from epidermis basal layer leading to achromic or hypochromic patches, and LS have been reported to be highly associated with autoimmune diseases and can co-occur (4).However Attili et al (5) reviewed 266 vitiligo cases and did not find association with LS . They suggest that association of vitiligo and LS may have been documented due to the clinical misdiagnosis of vitiligoid LS lesions as vitiligo.Several mechanisms of hypopigmentation in LSA have been proposed as decreased melanin production, block in transfer of melanosomes to keratinocytes and melanocyte loss. Melanocyte loss, as consequence of a lichenoid dermatitis triggering an autoimmune reaction to melanocytes, has been proposed as pathogenic connection of documented association of LS with vitiligo

The risk of malignancy in LS is small but if it occurs it tends to develop rapidly. Squamous cell carcinoma, and less commonly melanoma, basal cell carcinoma has been reported predominantly in patients with vulval LS (1)

In conclusion, patients with LS, especially women with LS, should be screened for other autoimmune diseases. Vitiligo must be differentiated from vitiligoid LS. Long-term follow-up is appropriate for patients with vulval LS

\section{REFERENCES}

[1] Neill S.M.,Lewis F.M., Tatnall F.M., Cox N.H. British Association of Dermatologists“ guidelines for the management of lichen sclerosus 2010. Br Association of Dermatologists 2010;163: 672-682

[2] Kreuter A, Kryvosheyeva Y, Terras S, Moritz R, Möllenhoff K, Altmeyer P, Scola N, Gambichler T. Association of autoimmune diseases with lichen sclerosus in 532 male and female patients. ActaDermVenereol 2013;93: 238-241

[3] Cooper SM, Ali I, Baldo M, Wojnarowska F. The association of lichen sclerosus and erosive lichen planus of the vulva with autoimmune disease: a case- control study. Arch Dermatol 2008; 144: $1432-1435$

[4] Weisber EL, Le LQ, Cohen JB. A case of simultaneous occurring lichen sclerosus and segmental vitíligo: conecting the underlying autoinmune pathogenesis. Int J Dermatol 2008; 47: 1053-1055

[5] Attili VR, Attili SK. Vitiligoid lichen sclerosus. A reappraisal. Indian J DermatolVenereolLeprol 2008; 74: 118-121 